\title{
Minor Effect of Antibiotic Pre-treatment on the Engraftment of Donor Microbiota in Fecal Transplantation in Mice
}

\author{
Tobias L. Freitag ${ }^{1,2+}$, Anna Hartikainen ${ }^{3+}$, Hanne Jouhten ${ }^{3}$, Cecilia Sahl2, Seppo Meri, ${ }^{1,2}$, \\ Veli-Jukka Anttila ${ }^{4}$, Eero Mattila ${ }^{4}$, Perttu Arkkila ${ }^{5}$, Jonna Jalanka ${ }^{3 *+}$ and \\ Reetta Satokari ${ }^{* *+}$ \\ ${ }_{1}^{1}$ Translational Immunology Research Program, Faculty of Medicine, University of Helsinki, Helsinki, Finland, ${ }^{2}$ Department \\ of Bacteriology and Immunology, Faculty of Medicine, University of Helsinki, Helsinki, Finland, ${ }^{3}$ Human Microbiome Research \\ Program, Faculty of Medicine, University of Helsinki, Helsinki, Finland, ${ }^{4}$ Department of Infectious Disease, Helsinki University \\ Central Hospital, Helsinki, Finland, ${ }^{5}$ Department of Gastroenterology, Helsinki University Central Hospital, Helsinki, Finland
}

\section{OPEN ACCESS}

Edited by:

Benjamin P. Willing,

University of Alberta, Canada

Reviewed by:

Alinne Castro,

Universidade Católica Dom Bosco

(UCDB), Brazil

Karen Madsen,

University of Alberta, Canada

*Correspondence:

Jonna Jalanka

jonna.jalanka@helsinki.fi

Reetta Satokari

reetta.satokari@helsinki.fi

†These authors have contributed equally to this work

Specialty section:

This article was submitted to

Microbial Symbioses,

a section of the journal

Frontiers in Microbiology

Received: 05 July 2019 Accepted: 05 November 2019 Published: 21 November 2019

Citation:

Freitag TL, Hartikainen A, Jouhten H, Sahl C, Meri S, Anttila V-J, Mattila E, Arkkila P, Jalanka J and

Satokari R (2019) Minor Effect

of Antibiotic Pre-treatment on the Engraftment of Donor Microbiota

in Fecal Transplantation in Mice.

Front. Microbiol. 10:2685.

doi: 10.3389/fmicb.2019.02685
Fecal microbiota transplantation (FMT) is an effective therapy for recurrent Clostridioides difficile infection $(\mathrm{rCDI})$ and is also considered a potential treatment for a wide range of intestinal and systemic diseases. FMT corrects the microbial dysbiosis associated with $\mathrm{rCDI}$, and the engraftment of donor microbiota is likely to play a key role in treatment efficacy. For disease indications other than rCDI, FMT treatment efficacy has been moderate. This may be partly due to stronger resilience of resident host microbiota in patients who do not suffer from rCDI. In rCDI, patients typically have undergone several antibiotic treatments prior to FMT, depleting the microbiota. In this study, we addressed the effect of broad-spectrum antibiotics (Ab) as a pretreatment to FMT on the engraftment of donor microbiota in recipients. We conducted a pre-clinical study of FMT between two healthy mouse strains, Balb/c as donors and C57BL/6 as recipients, to perform FMT within the same species and to mimic interindividual FMT between human donors and patients. Microbiota composition was assessed with high-throughput $16 \mathrm{~S}$ rDNA amplicon sequencing. The microbiota of Balb/c and C57BL/6 mice differed significantly, which allowed for the assessment of microbiota transplantation from the donor strain to the recipient. Our results showed that Ab-treatment depleted microbiota in C57BL/6 recipient mice prior to FMT. The diversity of microbiota did not recover spontaneously to baseline levels during 8 weeks after Ab-treatment, but was restored already at 2 weeks in mice receiving FMT. Interestingly, pre-treatment with antibiotics prior to FMT did not increase the overall similarity of the recipient's microbiota to that of the donor's, as compared with mice receiving FMT without $\mathrm{Ab}$-treatment. Pre-treatment with $\mathrm{Ab}$ improved the establishment of only a few donor-derived taxa, such as Bifidobacterium, in the recipients, thus having a minor effect on the engraftment of donor microbiota in FMT. In conclusion, pre-treatment with broadspectrum antibiotics did not improve the overall engraftment of donor microbiota, but did improve the engraftment of specific taxa. These results may inform future therapeutic studies of FMT.

Keywords: microbiota, fecal transfer, antibiotics, dysbiosis, bifidobacteria, microbiome 


\section{INTRODUCTION}

Fecal microbiota transplantation is an effective and curative treatment for rCDI (Mattila et al., 2012; van Nood et al., 2013; Quraishi et al., 2017), also eliminating rCDI-associated dysbiosis (Fuentes et al., 2014; Jalanka et al., 2016). Randomized clinical trials using FMT as a treatment for other intestinal diseases, such as UC (Narula et al., 2017) or IBS (Xu et al., 2019), have, however, had moderate or little success. One difference in the treatment regimens utilizing FMT for rCDI compared with other diseases is the use of antibiotics. Usually, rCDI patients are treated with antibiotics (e.g., vancomycin or metronidazole) prior to FMT, but antibiotics are rarely used before FMT for other indications. Generally, patients will undergo bowel lavage, provided that FMT is administered via colonoscopy.

Antibiotics are known to modulate the composition of the intestinal microbiota in both quantitative and qualitative terms (Dethlefsen and Relman, 2011; Ianiro et al., 2016), and their therapeutic efficacy has been demonstrated in non-infectious diseases such as IBS and hepatic encephalopathy (Ianiro et al., 2016). In addition to antibiotics, bowel lavage will reduce the number of intestinal bacteria and affect the microbial ecosystem temporarily (Morotomi et al., 1989; Jalanka et al., 2015). Although recent studies have shown that donor microbiota and specific donor-derived strains colonize the recipient after FMT (Jalanka et al., 2016; Li et al., 2016; Allegretti et al., 2019), it remains unclear whether donor strain engraftment is a prerequisite for the therapeutic efficacy of FMT in rCDI. In published studies of FMT for both rCDI and UC, similarity between donor and recipient microbiota profiles was higher in treatment responders (Moayyedi et al., 2015; Rossen et al., 2015; Staley et al., 2016), arguing for a critical role of the donor strain engraftment in treatment efficacy in patients undergoing FMT.

It has been hypothesized that antibiotic pre-conditioning may lead to a more effective transfer of the donor microbiota to the recipient, resulting in a higher similarity between donor and recipient microbiota profiles after FMT. If this were true, antibiotic pre-conditioning might lead to better treatment outcomes in patients undergoing FMT. Few previous experimental studies have also addressed the contribution of antibiotic pre-treatment before FMT to donor microbiota engraftment efficacy. Three studies that analyzed the effect of antibiotics before transplantation of microbiota from human feces into mice, thus addressing the interspecies transfer, produced divergent findings, suggesting that broad-spectrum antibiotics may support microbiota engraftment across the species barrier (Wos-Oxley et al., 2012; Ji et al., 2017; Staley et al., 2017). Only two studies analyzed the effects of broadspectrum antibiotics before FMT between different strains of the same mammalian species. In rats, antibiotic pre-treatment did not improve the engraftment of donor's microbiota (Manichanh et al., 2010), whereas in juvenile mice antibiotics in combination

Abbreviations: Ab, antibiotic; ANOVA, analysis of variance; BL, baseline; FMT, fecal microbiota transplantation; IBS, irritable bowel syndrome; Lav, lavage; PBS, phosphate-buffered saline; PCoA, principal coordinates analysis; PERMANOVA, permutational multivariate analysis of variance; $\mathrm{rCDI}$, recurrent Clostridioides difficile infection; UC, ulcerative colitis. with bowel lavage enhanced donor microbiota engraftment (Le Roy et al., 2018). However, this effect appeared to depend mainly on bowel lavage.

To further test the hypothesis that antibiotic pre-treatment before the administration of FMT will enhance microbiota engraftment, we performed an experimental study of FMT between adult mice from two different inbred strains, Balb/c as the donor and $\mathrm{C} 57 \mathrm{BL} / 6$ as the recipient, mimicking interindividual differences between human donors and patients. We compared microbiota changes in recipient mice pre-treated with a cocktail of broad-spectrum antibiotics (ciprofloxacin, metronidazole, and vancomycin) and receiving FMT after bowel lavage with the changes in recipient mice treated with bowel lavage and FMT, or FMT alone. These results were compared with those of a group of recipient mice receiving Ab-treatment and sham FMT, to analyze the compositional changes of recipient microbiota introduced by antibiotics.

\section{MATERIALS AND METHODS}

\section{Mice and Treatments}

Four adult Balb/c female mice (FMT donors) were purchased from Envigo (Madison, WI, United States), maintained under specific pathogen-free conditions in ventilated/autoclaved cages, and fed sterilized chow (Teklad Global 16\% Rodent Diet 2916C, Envigo). A colony of C57BL/6 mice (FMT recipients), originally purchased from The Jackson Laboratory (Bar Harbor, ME, United States), were maintained in the same facility and under the same conditions, but in strict isolation from the Balb/c mice. One week after arrival of the Balb/c mice at the facility, cages were changed in the evening, and stool pellets were collected from all four stool donors next morning. The stool material was stored as described previously (Satokari et al., 2015). Briefly, $500 \mathrm{mg}$ of stool was suspended in $5 \mathrm{ml}$ of sterile PBS, filtered through an autoclaved metal sieve, and $5 \mathrm{ml}$ of suspensions containing $10 \%$ glycerol were stored at $-80^{\circ} \mathrm{C}$ until use. Donor mice were sacrificed after stool sample collection, and coecum and colon segments were frozen at $-80^{\circ} \mathrm{C}$. Two samples of pooled donor feces were used for microbiota analysis.

Four groups of female C57BL/6 mice, maintained under the conditions specified above, were matched from 7 litters (3-5 months old; $n=6-7$ ), following collection of stool samples from all individuals on day 1 (baseline, BL; collection as above, Figure 1). On the same day, the drinking water for the two antibiotic pre-treated groups was supplemented with $660 \mathrm{mg} / \mathrm{l}$ ciprofloxacin (Yliopiston Apteekki, Helsinki, Finland), 1 g/l metronidazole (Sigma-Aldrich, St. Louis, MO, United States), and $500 \mathrm{mg} / \mathrm{l}$ vancomycin (Orion Pharma, Espoo, Finland). On day 10, antibiotic treatment was stopped. Stool samples were collected from all individuals on day 11 (time point preFMT). On the same day, three of the groups were treated with $200 \mu \mathrm{l}$ of colonsteril by oral gavage, containing $12 \mathrm{mg}$ of macrogol 4000, prepared according to the manufacturer's instructions (Orion Pharma, Espoo, Finland). Mice from all of the groups received one injection intraperitoneally of $2.5 \mathrm{mg}$ of ranitidine hydrochloride in $100 \mu \mathrm{l}$ volume (GlaxoSmithKline, 

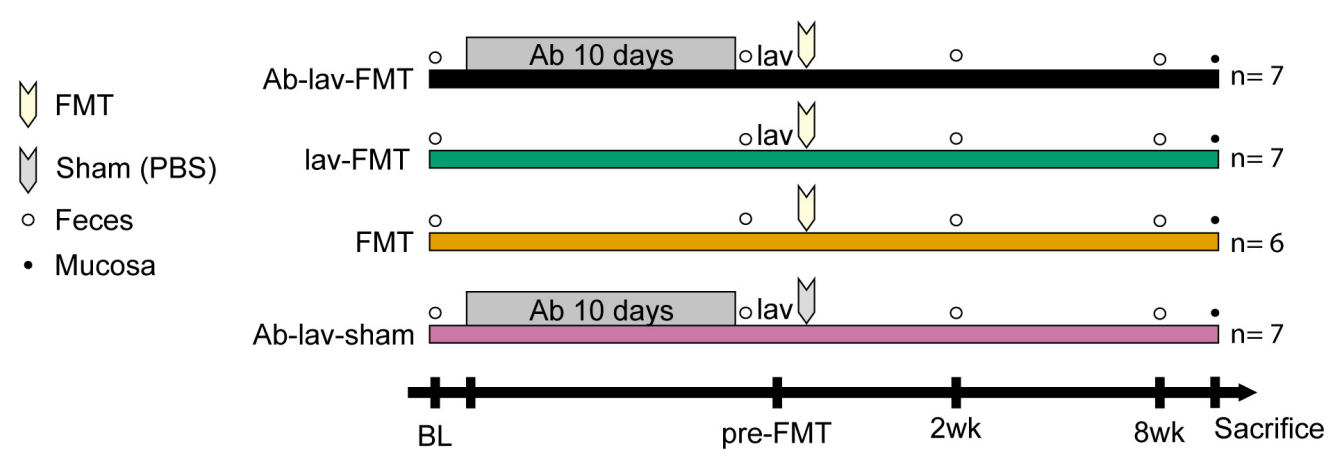

FIGURE 1 | Study design showing the treatment groups of recipient mice. After sampling of feces at baseline (recipients before treatments, BL) from four groups of mice, two groups of mice were pre-treated for 10 days with an antibiotic cocktail (Ab), consisting of 660 mg/l ciprofloxacin, 1 g/l metronidazole, and 500 mg/l vancomycin in drinking water. Two groups of mice received fecal microbiota transplantation (FMT) after bowel lavage (lav), one group received FMT without lav, and one groups received sham FMT with phosphate-buffered saline (PBS) after lav. The time points of fecal (empty bullets) or mucosal (full bullets) samplings are indicated.

Zeist, Netherlands). Five hours later, $200 \mu \mathrm{l}$ of pooled, thawed stool suspension (equal to $20 \mathrm{mg}$ of stool) from Balb/c donor mice was administered by oral gavage to three groups of mice, while the remaining sham-FMT group received $200 \mu \mathrm{l}$ of $10 \%$ glycerol in PBS. On days 25 (time point 2 weeks) and 71 (time point 8 weeks), stool samples were collected from all individuals and stored at $-80^{\circ} \mathrm{C}$ for microbiota analysis. On day 72 , mice were injected with ketamine/xylazine, and exsanguinated by retroorbital bleeding. Coecum and colon segments were collected and frozen at $-80^{\circ} \mathrm{C}$ for microbiota analysis. All animal procedures were approved by the Southern Finnish State Administrative Agency (ESAVI/1286/04.10.07/2016).

\section{Fecal Sample DNA Extraction}

A previously described, a validated method for fecal sample microbial DNA extraction was used (Salonen et al., 2010; Santiago et al., 2014). Repeated bead beating was used as a pretreatment, followed by high-throughput DNA extraction using KingFisher Flex 96 (Thermo Fisher Scientific, Vantaa, Finland). Extracted DNA was purified with QIAamp DNA Mini-kit (Qiagen, Hilden, Germany), and concentration measured using the Quant-iT ${ }^{\mathrm{TM}}$ PicoGreen $^{\mathrm{TM}}$ dsDNA Assay Kit (Invitrogen ${ }^{\mathrm{TM}}$, Eugene, OR, United States).

\section{Biopsy DNA Extraction}

DNA from the biopsy samples was extracted using a previously described method (Kalliomäki et al., 2012). In short, enzymatic lysis and repeated bead beating were used to disrupt microbial cells. DNA was extracted using the Qiagen DNeasy minispin column (Qiagen, Hilden, Germany), according to the manufacturer's instructions for Gram-positive bacteria. DNA concentration was measured using the Quant-iT ${ }^{\mathrm{TM}}$ PicoGreen ${ }^{\mathrm{TM}}$ dsDNA Assay Kit (Invitrogen ${ }^{\mathrm{TM}}$, Eugene, OR, United States).

\section{Microbial DNA Compositional Analysis}

Microbial composition in fecal samples was analyzed by sequencing the hypervariable V3-V4 region of the 16S rRNA gene with Illumina MiSeq (Klindworth et al., 2013). For the analysis of mucosal microbiota, full-length 16S rRNA PCR was conducted first to enrich the microbial DNA in biopsy DNA samples. Briefly, 1500 bp amplicon was generated by using the previously designed primers (Wang et al., 2002) and by using the following amplification conditions: one cycle of $95^{\circ} \mathrm{C}$ for $2 \mathrm{~min}$, followed by 20 cycles of $95^{\circ} \mathrm{C}$ for $20 \mathrm{~s}, 55^{\circ} \mathrm{C}$ for $20 \mathrm{~s}, 72^{\circ} \mathrm{C}$ for $90 \mathrm{~s}$, and a final extension cycle of $72^{\circ} \mathrm{C}$ for $5 \mathrm{~min}$. PCR products were purified using the QIAquick ${ }^{\circledR}$ PCR Purification Kit (Qiagen, Hilden, Germany) according to the manufacturer's instructions. The purified PCR product was used as the template for 16S rRNA gene V3-V4 region sequencing similarly to the fecal samples by using the MiSeq platform. The analysis was successful for 102 of 108 fecal samples and for 36 of 54 biopsy samples. The data were deposited to the European Nucleotide Archive (PRJEB33286).

For processing and analyzing the reads, $\mathrm{R}$ package mare was used (Korpela, 2016). Forward reads trimmed to $150 \mathrm{nt}$ were used for the analysis. This approach has been previously observed to produce highly reliable results when using artificial communities of known composition (Korpela, 2016). To exclude errors and chimeras, rare reads were eliminated by using a quality filter for minimal read abundance of $0.001 \%$, and reads were chimera filtered. After pre-processing, the average read number was 49,607 per sample (ranging from 6,249 to 335,038, median 46,629). Taxonomic annotation was made by using the USEARCH (version 8.1.1756) and SILVA 16S rRNA reference database version 115 (Quast et al., 2013).

\section{Statistical Methods}

For statistical analysis, $\mathrm{R}$ package mare was used (Korpela, 2016). It uses tools from $R$ packages vegan (Oksanen et al., 2019), MASS (Venables and Ripley, 2002), and nlme (Pinheiro et al., 2019). Genus-level data were used for the statistical testing. To test differences in the bacterial abundance between study groups at certain time points, generalized linear mixed models using the function glm.nb from package MASS were used. If there was a failure in fulfilling the model assumptions, alternatively generalized least squares models were used. The read number for each sample was used 
as an offset. The obtained $p$-values were corrected using multiple testing with false discovery rate approach, and FDRadjusted $p$-values ( $q$-values) below 0.05 were considered to be significant. Principal co-ordinate analysis was used to visualize microbial composition at different time points and study groups. The function used utilizes $\mathrm{R}$ package vegan and Bray-Curtis dissimilarities. Moreover, to estimate the grouping effect on overall microbiota composition, adonis function (PERMANOVA) in $\mathrm{R}$ package vegan was used. Additionally, ANOVA followed by Tukey's HSD post hoc test or Student's $t$-test was used for evaluating statistical significance in normally distributed data. Normality of the data was tested by using the Shapiro-Wilk test. Similarity between the samples was calculated with Spearman's correlation $(\rho)$. OriginPro 2018 (version b9.5.5.409 Academic) and CorelDRAW (version 20.1.0.708) were used for visualizing the data.

\section{RESULTS}

The main aim of this study was to analyze the effect of antibiotic pre-treatment on microbiota engraftment after FMT. The study design is depicted in Figure 1. Briefly, we compared the microbial changes of the recipient mice pre-treated with antibiotics and receiving FMT after bowel lavage with the three different control groups. These included mice that had also received antibiotic pretreatment and sham FMT after the lavage, in addition to a group of mice treated with either bowel lavage and FMT or FMT alone.

\section{Fecal Microbial Populations in Donors and Recipients Differed at Baseline}

At baseline, the fecal microbiota in Balb/c donors differed significantly from that in C57BL/6 recipients (Figures 2A,B and Supplementary Figure S1A). The differences between these mouse strains explained $18 \%$ ( $p=0.002$, PERMANOVA) of the microbial variation, and the microbiota similarity between the recipients and donors, measured with Spearman's correlation, was on average $0.57 \pm 0.03$ (SD). At bacteria phylum level, the differences included higher levels of Firmicutes and Actinobacteria in donors (62.62 vs. $21.80 \%$, and 9.20 vs. $0.83 \%$, respectively, Figure 2B). Recipient mice had higher levels of Bacteroidetes and Verrucomicrobia (58.25 vs. $24.72 \%$, and 14.11 vs. $0.04 \%$, respectively). On average, there were $39.75 \pm 2.22$ genus-level taxa that had statistically different abundance (FDR-adjusted $p<0.05$ ) between donors and recipients (Supplementary Table S1). The observed microbiota differences between the mouse strains allowed for the assessment of microbiota engraftment from the donor strain to the recipient based on microbiota profiling.

\section{Effect of Antibiotic Treatment on Microbiota Composition}

We first assessed the effect of antibiotic treatment on the microbiota. In addition to the group that later received FMT after antibiotics pre-treatment (Ab-lav-FMT), a control group of mice that later received sham FMT was also pre-treated with antibiotics (Ab-lav-sham). The antibiotic treatment had a strong effect on microbiota, with $79 \%$ of the microbial variation between the study groups explained by the consumption of antibiotics $(p=0.01$, PERMANOVA, Figure 2C and Supplementary Figure S1B) at time point pre-FMT. Microbial diversity was decreased significantly in the antibiotic-treated animals compared with the untreated animals $\left(p=9.96 \times 10^{-12}\right.$, ANOVA, Tukey's post hoc, Figure 3A). The diversity did not recover to baseline levels in the Ab-lav-sham group during the follow-up period. This was reflected also in the similarity analysis, where the Ab-lav-sham group showed little similarity to their own baseline microbiota, both at 2 weeks $(\rho=0.60 \pm 0.08$, Figure 3B $)$ and at 8 weeks $(\rho=0.59 \pm 0.08$, Figure 3B) after sham FMT.

The microbial composition of animals treated with antibiotics was significantly reduced after the antibiotic treatment (at time point pre-FMT), and only 44 of the 117 genera detected at baseline were present in over $0.01 \%$ abundance (Supplementary Table S2). Here, the most abundant genuslevel taxa were Verrucomicrobium (84.78\%), Lactobacillus (6.70\%), uncultured member of Bacteroidetes $(2.38 \%)$, and Parabacteroides (1.63\%), representing on average $95.49 \%$ of all detected taxa (Supplementary Table S3).

\section{Effect of Antibiotic Pre-treatment on Microbiota Engraftment}

Next, we studied the recovery of antibiotics-depleted microbiota by FMT, and the differences in donor microbiota engraftment between the animals that received and those that did not receive antibiotics before FMT. The dramatic decrease in microbial diversity caused by the antibiotics was restored by FMT (Figure 3A). The increase in diversity was detected already 2 weeks after the treatment in the Ab-lav-FMT group, and it was sustained until the end of the study (Figure 3A). Compared with the control groups, diversity of the Ab-lav-FMT group was similar to the mice that were treated with FMT but did not receive antibiotics (FMT and lav-FMT) (Figure 3A). However, in the Ab-lav-sham group, diversity remained low at 2 and 8 weeks after sham FMT.

When visualizing compositional dissimilarity with PCoA, the donor's microbiota was found to cluster separately from that of FMT-treated animals. In addition, the samples from baseline and after FMT were mixed and did not show any significant shift toward the donor samples (Figure $\mathbf{4 A}$ and Supplementary Figure S1C). This observation was supported by the similarity analyses showing a higher correlation of the microbial composition in mice from all FMT groups with their own baseline $(\rho=0.77, \pm 0.02$, Figure 3B) than with donors $(\rho=0.68 \pm 0.02$, Figure 4B). In this study, the within-animal similarity in the C57BL/ 6 mice was $0.89 \pm 0.05$.

However, the similarity between donors and recipients was low at baseline $(\rho=0.57 \pm 0.03)$, and it increased significantly 2 weeks after FMT in the treated animals (Ablav-FMT: $19.21 \%$ increase, $p=2.73 \times 10^{-5}$; lav-FMT: $9.44 \%$ increase, $p=0.001$; FMT group: $12.89 \%$ increase, $p=0.0003$, ANOVA, Tukey's post hoc, Figure 4B). This increase in similarity was sustained until the end of the study, 8 weeks from 



FIGURE 2 | (A) PCoA of microbiota profiles at bacterial genus level from the study groups at baseline. (B) Fecal microbiota composition in donor and recipient mice at baseline. Relative abundance scaled to 100\%, and number of sequence reads marked with bullets. Within phyla, individual genera are shown with different shades, and the lightest color shows the combined abundance of the least abundant genera ( $<0.5 \%$ of total). (C) Microbiota composition of donor and recipient mice at other time points in the study.

FMT (Figure 4B). In contrast, in the Ab-lav-sham group the similarity to donors decreased from baseline due to the antibiotic treatment and did not recover in the follow-up time points at 2 weeks $\left(p=3.97 \times 10^{-4}\right.$, ANOVA, Tukey's post hoc) and 8 weeks $\left(p=1.71 \times 10^{-4}\right.$, ANOVA, Tukey's post hoc) after the sham FMT. In addition, the Ab-lav-sham group separated significantly from other treatment groups in PCoA analyses at the end of the study concerning both fecal ( $p=0.001$, PERMANOVA, Figure 4C and Supplementary Figure S1D) and mucosal ( $p=0.001$, PERMANOVA, Figure 4D and Supplementary Figure S2) microbiota composition. In summary, FMT restored the microbial diversity disrupted by antibiotics, but antibiotic pre-treatment did not increase the overall similarity between donor and recipient microbiota, as compared with control groups.

\section{Specific Genus-Level Differences in Microbial Composition After FMT Are Attributable to Antibiotic Pre-treatment}

To assess the magnitude of the changes introduced by the antibiotic pre-treatment, we determined the number of significantly differing taxa between the Ab-lav-FMT and other groups as well as the donors at different time points (Figure 5A). The obtained FDR-adjusted $p$-values were calculated using generalized linear mixed models. At baseline, the donors had the 

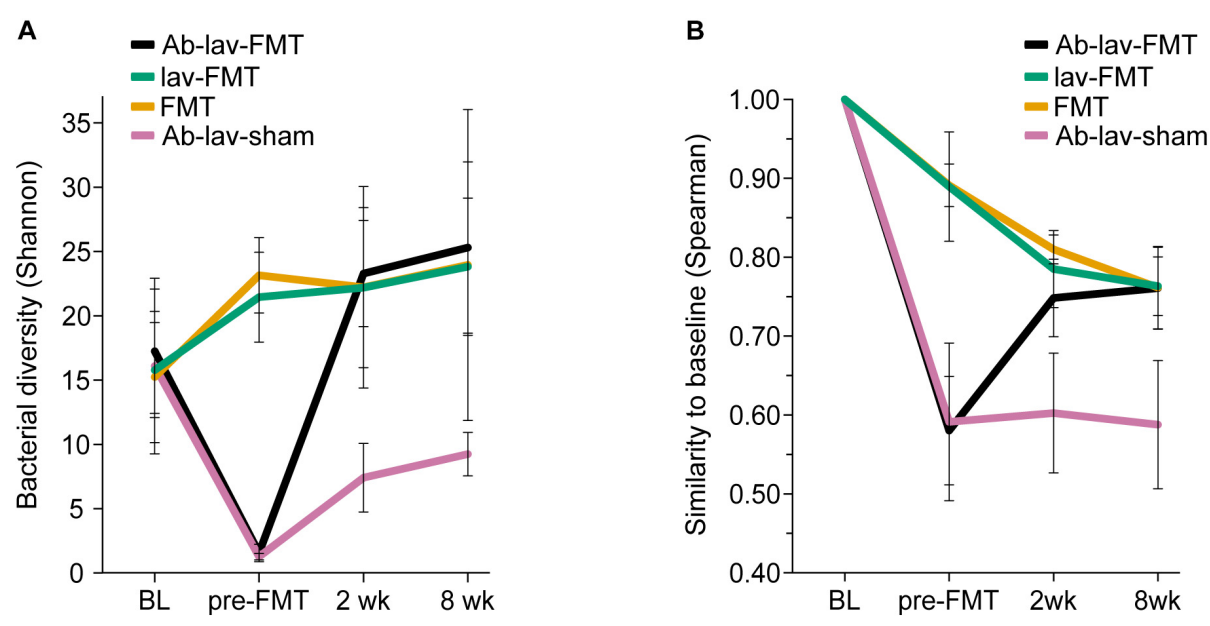

FIGURE 3 | (A) Bacterial diversity in different treatment groups across all time points. (B) Similarity between the baseline sample and consecutive samples in all study groups across all time points.

greatest number (41 of 117 detected) of differing taxa relative to the Ab-lav-FMT group, which is consistent with the mouse strain effect. After the antibiotic pre-treatment, the number of differing taxa increased sharply compared with the animals not treated with antibiotics. During the follow-up period the Ablav-FMT group remained relatively different from the other FMT-treated groups (19-32 of the genera showed a statistical difference), which remained highly similar to each other during the follow-up period (only 3-4 of the genera showed a statistical difference between lav-FMT and FMT groups, Supplementary Figures S3A-C). Throughout the study, the number of taxa differing between all recipient groups and the donors remained high (37-62 of the genera, Supplementary Figures S3A-D).

Next, we determined the number of genus-level taxa that were differentially engrafted in mice pre-treated with antibiotics compared with the control groups. This was done again by using a generalized linear mixed model to test the difference between study groups at both follow-up time points. Two weeks after FMT, there were 28 significantly different taxa between the Ab-lav-FMT and lav-FMT groups, and 32 between the Ab-lav-FMT and FMT groups. Antibiotic pre-treatment had a substantial effect on the engraftment since there were only three taxa with significantly different abundance 2 weeks after FMT between the lav-FMT and FMT groups (Supplementary Figures S3B,C). Thus, antibiotic pre-treatment had a detectable effect on the taxa level, but only a minor effect on overall microbiota engraftment after FMT.

To determine whether the antibiotic pre-treatment improved the engraftment of specific taxa, we compared the mean relative abundances of the significantly different taxa in different groups 2 weeks after FMT. Taxa that were detected in the donors but were absent in the Ab-lav-sham group and were more increased in group Ab-lav-FMT than in groups lav-FMT or FMT were deemed to have benefited in their engraftment from antibiotic pre-treatment. There were five genus-level taxa that matched these criteria: Bifidobacterium, Adlercreutzia, Enterorhabdus, Odoribacter, and Alkalibacterium (Supplementary Table S4).
At baseline, the genus Bifidobacterium was present in recipient mice on average at $0.13 \% \pm 0.001$ abundance, and in donors at $5.72 \% \pm 2.26$ abundance (Figure 5B). Two weeks after FMT, the bifidobacterial abundance in the lav-FMT and FMT groups remained low (on average $0.34 \% \pm 0.002$ ), whereas in the Ab-lavFMT group it increased significantly to the level of $2.25 \% \pm 0.014$ $\left(p=5.59 \times 10^{-9}\right)$. In this group, bifidobacterial abundance remained increased also at 8 weeks after FMT $(1.29 \% \pm 0.012$, $\left.p=6.02 \times 10^{-6}\right)$. Overall, the antibiotic pre-treatment had a detectable effect on the engraftment of specific genus-level taxa, most notably on the abundance of Bifidobacterium.

In a similar way, we sought bacterial taxa that were decreased after FMT in mice pre-treated with antibiotics, but were present in the other FMT groups on higher level 2 weeks after FMT-treatment. In total, eight genus-level taxa were significantly less abundant (FDR-adjusted $p$-value $<0.05$; Supplementary Table S4) in Ab-lav-FMT group than in other FMT-treated groups. Thus, antibiotic pre-treatment suppressed some taxa that were not re-introduced by FMT from the other mice strain. On the other hand, antibiotic treatment as such increased the relative abundance of three genus-level taxa, and Marvinbryantia, Stomatobaculum, and an uncultured member of Firmicutes (incertae sedis) increased in both antibiotic-treated groups, i.e., groups with and without FMT (Supplementary Table S4). Interestingly, the abundance of Verrucomicrobium was increased after the antibiotic pre-treatment (Figure 2C), was not detected in the donors, and the abundance of this taxa was decreased in all groups after FMT, but not after sham FMT (Supplementary Table S4).

\section{Species Richness in Donor and Recipient Mice}

At baseline, donors had significantly higher richness than recipient mice $\left(p=1.0 \times 10^{-7}\right.$, for all group comparisons, ANOVA, Tukey's post hoc, Supplementary Figure S4). The higher richness was transferred with FMT to all treated animals, 
A

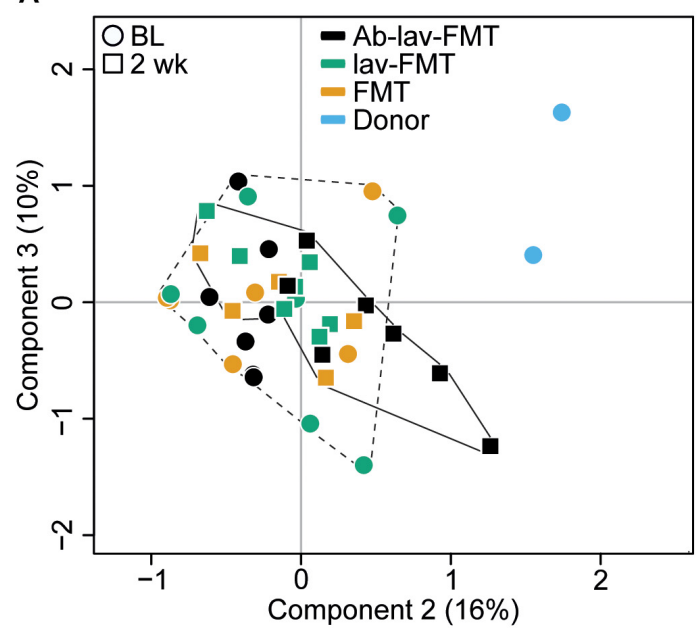

C

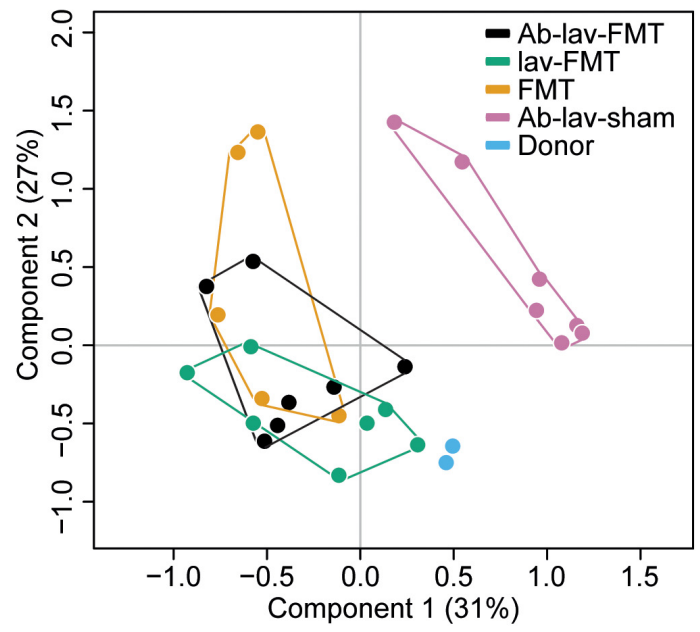

B

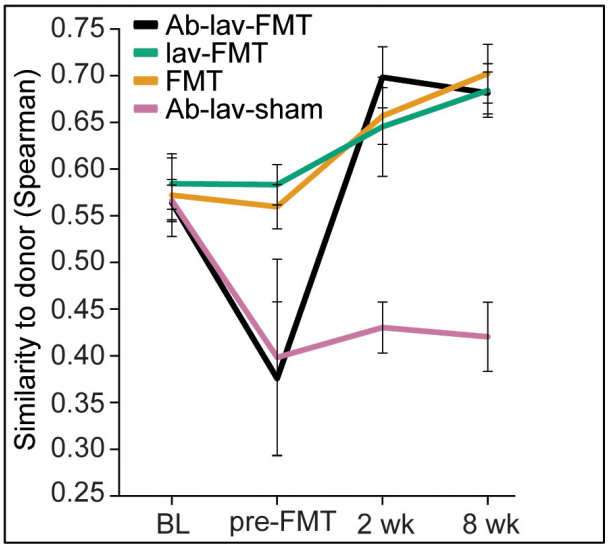

D



FIGURE 4 | (A) PCoA of fecal microbiota composition: effects of antibiotic pre-treatment and study time point. Ab-lav-sham group is excluded to visualize the distribution of the FMT group samples in more detail. (B) Microbial similarity to donor for different groups throughout the study. Similarity measured with Spearman correlation. (C) PCoA of fecal microbiota composition at 8 weeks after (sham) FMT: significant separation of Ab-lav-sham group from the other groups $(p=0.001$, PERMANOVA). (D) PCOA of mucosal microbiota composition at 8 weeks after (sham) FMT: significant effects of antibiotic treatment $(p=0.001$, PERMANOVA) and biopsy location $(p=0.001$, PERMANOVA).

where the richness remained high at the follow-up time points $(p<0.001$, ANOVA, Tukey's post hoc, Supplementary Figure S4). Thus, significant difference in richness between Ablav-FMT and other FMT-treated groups was not detected 2 or 8 weeks after FMT-treatment (comparison of Ab-lav-FMT and lav-FMT 2 weeks after FMT-treatment $p=0.99$, Ab-lav-FMT and FMT 2 weeks after FMT-treatment $p=0.98$ and comparison of Ab-lav-FMT and lav-FMT eight weeks after FMT-treatment $p=0.27$, Ab-lav-FMT and FMT 2 weeks after FMT-treatment $p=0.99$, ANOVA, Tukey's post hoc).

\section{Microbial Composition in Colonic Mucosa and Length of Colon}

At the end of the study, the coecum microbiota of the FMTtreated mice was composed mainly of Firmicutes $(89.50 \% \pm 0.83)$ and Bacteroidetes $(6.08 \% \pm 0.50)$, whereas microbiota in the sham-FMT group was dominated by only Firmicutes (96.99\%) (Supplementary Figure S5). Similarly, mainly Firmicutes and Bacteroidetes were detected in the distal colon of the FMT-treated animals $(82.90 \% \pm 4.51$ and $11.93 \% \pm 4.11$, respectively), but in the sham-FMT group the microbiota was dominated by Firmicutes (68.61\%) and Deferribacteres (29.03\%) (Supplementary Figure S5).

In PCoA, mucosal samples from donors and all FMT-treated animals clustered together, whereas the Ab-lav-sham group separated from the others ( $p=0.001$, PERMANOVA, Figure 4D and Supplementary Figure S2). Moreover, similarity to donor microbiota was low for the Ab-lav-sham group (Supplementary Figure S6). In contrast, all of the FMT groups showed high similarity to donor microbiota (average $\rho=0.78 \pm 0.03$ ), but the highest similarity was observed in the Ab-lav-FMT group 
A

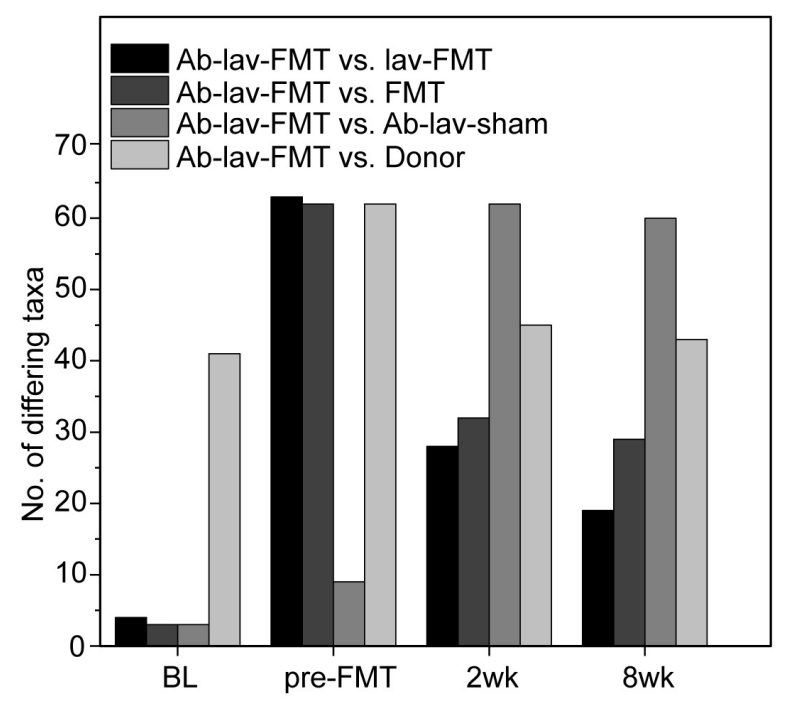

B

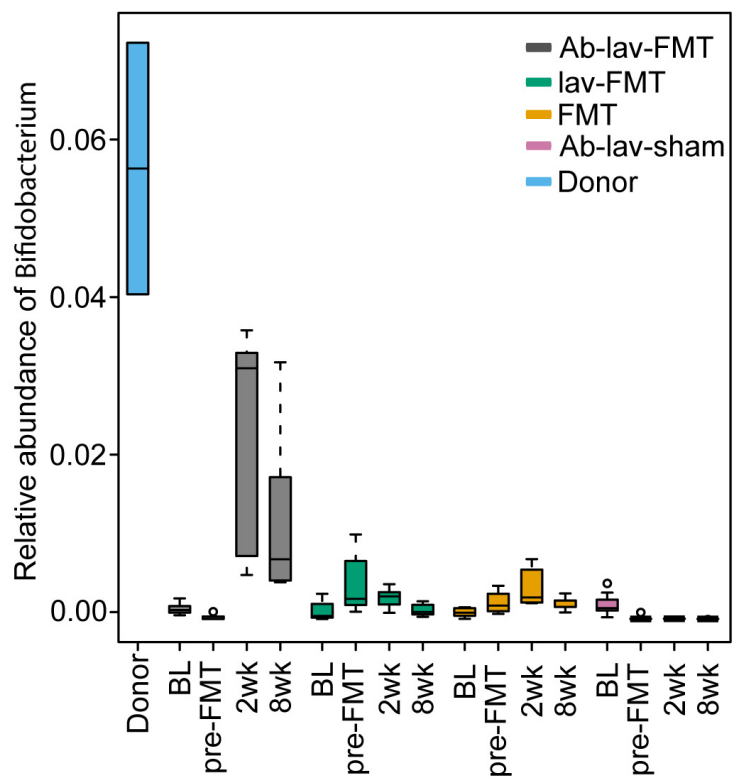

FIGURE 5 | (A) Number of genus-level bacterial taxa that differed in relative abundance (FDR-adjusted $p<0.05$ ) between the Ab-lav-FMT group versus all other groups, or versus donor, at four different time points. (B) Relative abundance of genus Bifidobacterium, demonstrating higher levels in Balb/c donor than in C57BL/6 recipient mice. Relative abundance increased in the Ab-lav-FMT group at 2 and 8 weeks after FMT compared with baseline $\left(p=5.59 \times 10^{-9}\right.$ and $6.02 \times 10^{-6}$, respectively), and also compared with other groups at 2 weeks after FMT (FDR-adjusted $p<0.05)$. On the other hand, levels of Bifidobacterium were decreased in the Ab-lav-sham group after antibiotic treatment and remained low until 8 weeks after sham FMT.

$(\rho=0.80 \pm 0.02)$. Thus, colonic microbiota in the FMT groups resembled those of the donors, whereas the Ab-lav-sham group had clearly distinct colonic microbiota profiles.

Colon length is known to differ between different mouse strains and/or colonies, and previous studies have demonstrated that $\mathrm{Balb} / \mathrm{c}$ mice have greater colon length than $\mathrm{C} 57 \mathrm{BL} / 6$ mice (Qualls et al., 2006). A comparison of colon length in the four C57BL/6 recipient groups showed that mice treated with antibiotics, lavage, and FMT had significantly longer colons than mice from all other groups combined, resembling the reported Balb/c donor phenotype (Figure 6, $p=0.02$, Student's $t$-test).

\section{DISCUSSION}

In this study, we assessed the effect of antibiotic pre-treatment on the engraftment of donor microbiota in FMT. Balb/c mice were used as FMT donors and C57BL/6 mice as the FMT recipient strain. At baseline, microbiota composition and richness differed between donors and recipients. As expected, pre-treatment with ciprofloxacin, metronidazole, and vancomycin significantly decreased the bacterial diversity. After FMT, the diversity, species richness, and similarity to donor microbiota rose in the Ab-treated group, but the same levels were also reached in the other FMT groups. Nonetheless, we found effects of the antibiotic pre-treatment on the engraftment of specific donor microbiota to recipient mice. Interestingly, abundance of a number of genus-level taxa, including Bifidobacterium, was significantly higher in the Ab-treated group 2 weeks after FMT than in the other study groups. Moreover, colon length in the Ab-treated group was significantly longer than in other recipient mice.

Our results demonstrating differences at baseline between two mouse strains are in line with those observed in an earlier metagenomic study, which examined five factors, including




mouse strain and provider, affecting the composition of the mouse gut microbiome (Xiao et al., 2015). In another study, higher richness of donor stool relative to recipient stool was seen as a positive predictor of microbiota engraftment after FMT (Ericsson et al., 2017). In our study, donors had significantly higher richness than recipients, indicating that conditions for donor microbiota engraftment were favorable. Overall, the results suggested that higher species richness was transferred to FMT recipients, but antibiotic pre-treatment offered no significant benefit.

The antibiotic cocktail (ciprofloxacin, metronidazole, and vancomycin) used in this study was active against a broad spectrum of Gram-negative, Gram-positive, and anaerobic bacteria and was regarded as suitable for clinical application. As expected, the pre-treatment decreased diversity and reduced the number of detected taxa. These findings are consistent with those of Dethlefsen and Relman (2011), who reported a shift in the distal gut microbial composition and a decrease of diversity after two courses of ciprofloxacin. In the current study, at genus level, taxa belonging to Verrucomicrobium, Lactobacillus, and Parabacteroides dominated the composition in the recipient mice after pre-treatment with antibiotics. The genera Lactobacillus and Parabacteroides were detected in both groups treated with antibiotics until the end of the study, whereas the abundance of Verrucomicrobium decreased after FMT in animals treated with antibiotics. Many Lactobacillus spp. are known to harbor resistance genes to vancomycin and ciprofloxacin or to have a resistant phenotype (Campedelli et al., 2019), and members of the Bacteroides fragilis group, including Parabacteroides distasonis, are among the anaerobes least susceptible to antibiotics (Schuetz, 2014). Therefore, it is not surprising that Lactobacillus and Parabacteroides were found to prevail after the antibiotic treatment. Verrucomicrobia, on the other hand, are a special phylum of bacteria that have complex endomembrane structures and a compartmentalized cell plan (Lee et al., 2009), which may account for their resistance against antibiotics. While we are unaware of the traits that conferred resistance of Verrucomicrobium to the antibiotics applied, its high abundance in Ab-treated animals indicates effective mechanisms. Notably, antibiotic resistance of specific bacteria by deactivation mechanism may give collective resistance also to other members of the microbial community (Sorg et al., 2016). Indeed, in addition to the above-mentioned three taxa, a number of other bacteria were also present in low amounts in the Ab-treated mice, and these may represent such co-benefiting taxa.

Although in our study the FMT restored microbial diversity after antibiotic pre-treatment, the results did not show a significant increase in overall similarity to donor microbial composition compared with animals not treated with antibiotics. Furthermore, the similarity analyses showed a higher correlation of the microbial composition in mice from all FMT groups with their own baseline, rather than with the donor communities, indicating resilience of the host microbiota. Therefore, the results of this study of FMT between two different inbred strains of mice did not support the main hypothesis that antibiotic pre-conditioning will lead to a more effective transfer of donor microbiota to the recipient as a whole. Nevertheless, specific taxa were shown to benefit from the antibiotic pre-treatment.

Here we showed that there were detectable effects on the abundance of specific bacterial taxa after the FMT. Five presumably Balb/c donor-derived genus-level taxa, including Bifidobacterium, were more abundant in the stool of recipients that had been pre-treated with antibiotics. In this group, bifidobacterial abundance remained increased until the end of the study. Since bifidobacteria are nonpathogenic, generally regarded as safe, and also used as probiotics, their promotion by antibiotic pre-treatment may be of interest for FMT, particularly as they are also known to exert anti-inflammatory and epithelium-reinforcing actions (Hiippala et al., 2018). Interestingly, a recent study in humans reported that Bifidobacterium was more abundant after FMT following pre-treatment with ciprofloxacin, although this was not compared with FMT without antibiotic pre-treatment (Kang et al., 2017). Furthermore, the colon length of mice pre-treated with antibiotics before FMT was increased at the end of the study relative to all other groups, indicating that the differences in microbiota composition may have had physiological effects, affecting the mouse phenotype.

Previous studies have produced divergent results regarding the effects of bowel cleansing prior to microbiota engraftment. One study demonstrated a positive effect of bowel cleansing (Le Roy et al., 2018), while another study found no impact of lavage alone, but a combined effect with lavage and antibiotics (Ji et al., 2017). While our study primarily addressed the effects of antibiotic pretreatment on donor microbiota engraftment, group comparisons suggested no improvement of engraftment by bowel lavage versus no bowel lavage. However, these results should be interpreted with caution because we had chosen a lower laxative dose for mice than the studies mentioned above. In humans, there is evidence that the effect on microbial composition is larger when higher amounts of laxative are used (Jalanka et al., 2015). Also, a recent rodent study showed that a high dose of purging agent introduced a significant reduction in bacterial load, resulting in better microbial engraftment (Wrzosek et al., 2018). Thus, an efficient lavage could provide some advantage for FMT, and attention should be paid to the lavage regimes in future studies.

In summary, we showed that in FMT between two different mice strains, mimicking the clinical settings, pretreating the mice with broad-spectrum antibiotics had only a minor effect on donor microbiota engraftment. One of the observations was better engraftment of the genus Bifidobacterium with antibiotic pre-treatment, which may offer potential benefits in future therapeutic studies. This was supported by the finding that colon length was greater in animals pre-treated with antibiotics, thereby mimicking the donor strain. Since the resilience of the recipient's microbiota appeared to limit the engraftment, a potential use for antibiotics before FMT may be the modulation of recipient microbiota profiles. Recipient 
microbiota modulation by antibiotic pre-treatment may act synergistically with donor-derived microbiota in resolving dysbiosis in human diseases.

\section{DATA AVAILABILITY STATEMENT}

The raw sequencing data for this study can be found in the European Nucleotide Archive (PRJEB33286).

\section{ETHICS STATEMENT}

The animal study was reviewed and approved by the Southern Finnish State Administrative Agency (ESAVI/1286/ 04.10.07/2016).

\section{AUTHOR CONTRIBUTIONS}

TF, SM, V-JA, EM, PA, and RS conceived and designed the study. JJ contributed to conception and design of the study. TF, JJ, $\mathrm{AH}, \mathrm{HJ}$, and CS performed animal work and laboratory analysis. $\mathrm{AH}$ and JJ performed the statistical analysis. TF, AH, JJ, and RS interpreted the results and wrote the first draft of the manuscript.

\section{REFERENCES}

Allegretti, J. R., Kassam, Z., Carrellas, M., Mullish, B. H., Marchesi, J. R., Pechlivanis, A., et al. (2019). Fecal microbiota transplantation in patients with primary sclerosing cholangitis: a pilot clinical trial. Am. J. Gastroenterol. 114, 1071-1079. doi: 10.14309/ajg.0000000000000115

Campedelli, I., Mathur, H., Salvetti, E., Clarke, S., Rea, M. C., Torriani, S., et al. (2019). Genus-wide assessment of antibiotic resistance in Lactobacillus spp. Appl. Environ. Microbiol. 85:e1738-18. doi: 10.1128/aem.017 38-18

Dethlefsen, L., and Relman, D. A. (2011). Incomplete recovery and individualized responses of the human distal gut microbiota to repeated antibiotic perturbation. Proc. Natl. Acad. Sci. U.S.A. 108(Suppl. 1), 4554-4561. doi: 10. 1073/pnas.1000087107

Ericsson, A. C., Personett, A. R., Turner, G., Dorfmeyer, R. A., and Franklin, C. L. (2017). Variable colonization after reciprocal fecal microbiota transfer between mice with low and high richness microbiota. Front. Microbiol. 8:196. doi: $10.3389 /$ fmicb.2017.00196

Fuentes, S., van Nood, E., Tims, S., Heikamp-de Jong, I., ter Braak, C. J., Keller, J. J., et al. (2014). Reset of a critically disturbed microbial ecosystem: faecal transplant in recurrent Clostridium difficile infection. ISME J. 8, 1621-1633. doi: 10.1038 /ismej.2014.13

Hiippala, K., Jouhten, H., Ronkainen, A., Hartikainen, A., Kainulainen, V., Jalanka, J., et al. (2018). The potential of gut commensals in reinforcing intestinal barrier function and alleviating inflammation. Nutrients 10:E988. doi: 10.3390/ nu10080988

Ianiro, G., Tilg, H., and Gasbarrini, A. (2016). Antibiotics as deep modulators of gut microbiota: between good and evil. Gut 65, 1906-1915. doi: 10.1136/gutjnl2016-312297

Jalanka, J., Mattila, E., Jouhten, H., Hartman, J., de Vos, W. M., Arkkila, P., et al. (2016). Long-term effects on luminal and mucosal microbiota and commonly acquired taxa in faecal microbiota transplantation for recurrent Clostridium difficile infection. BMC Med. 14:155. doi: 10.1186/s12916-016-0698-z

Jalanka, J., Salonen, A., Salojarvi, J., Ritari, J., Immonen, O., Marciani, L., et al. (2015). Effects of bowel cleansing on the intestinal microbiota. Gut 64, 1562 1568. doi: 10.1136/gutjnl-2014-307240
All authors contributed to manuscript revision and read and approved the submitted version.

\section{FUNDING}

This work was supported by the Sigrid Jusélius Foundation to RS and Academy of Finland grants (316338 to JJ and 292393 to SM).

\section{ACKNOWLEDGMENTS}

The sequencing was performed at the core facility of the Institute for Molecular Medicine Finland in Helsinki, Finland. We thank Mari Elemo at the Laboratory Animal Center of the University of Helsinki for performing oral gavage in mice for bowel lavage and FMT.

\section{SUPPLEMENTARY MATERIAL}

The Supplementary Material for this article can be found online at: https://www.frontiersin.org/articles/10.3389/fmicb. 2019.02685/full\#supplementary-material

Ji, S. K., Yan, H., Jiang, T., Guo, C. Y., Liu, J. J., Dong, S. Z., et al. (2017) Preparing the Gut with antibiotics enhances gut microbiota reprogramming efficiency by promoting xenomicrobiota colonization. Front. Microbiol. 8:1208. doi: $10.3389 /$ fmicb.2017.01208

Kalliomäki, M., Satokari, R., Lähteenoja, H., Vähämiko, S., Grönlund, J., Routi, T., et al. (2012). Expression of microbiota, toll-like receptors, and their regulators in the small intestinal mucosa in celiac disease. J. Pediatr. Gastroenterol. Nutr. 54, 727-732. doi: 10.1097/MPG.0b013e318241cfa8

Kang, D. W., Adams, J. B., Gregory, A. C., Borody, T., Chittick, L., Fasano, A., et al. (2017). Microbiota transfer therapy alters gut ecosystem and improves gastrointestinal and autism symptoms: an open-label study. Microbiome 5:10. doi: 10.1186/s40168-016-0225-7

Klindworth, A., Pruesse, E., Schweer, T., Peplies, J., Quast, C., Horn, M., et al. (2013). Evaluation of general 16S ribosomal RNA gene PCR primers for classical and next-generation sequencing-based diversity studies. Nucleic Acids Res. 41:e1. doi: 10.1093/nar/gks808

Korpela, K. (2016). Microbiota Analysis in R Easily. Available at: https://github. com/katrikorpela/mare (Accessed June 14, 2019).

Le Roy, T., Debédat, J., Marquet, F., Da-Cunha, C., Ichou, F., Guerre-Millo, M., et al. (2018). Comparative evaluation of microbiota engraftment following fecal microbiota transfer in mice models: age, kinetic and microbial status matter. Front. Microbiol. 9:3289. doi: 10.3389/fmicb.2018.03289

Lee, K. C., Webb, R. I., Janssen, P. H., Sangwan, P., Romeo, T., Staley, J. T., et al. (2009). Phylum Verrucomicrobia representatives share a compartmentalized cell plan with members of bacterial phylum Planctomycetes. BMC Microbiol. 9:5. doi: 10.1186/1471-2180-9-5

Li, S. S., Zhu, A., Benes, V., Costea, P. I., Hercog, R., Hildebrand, F., et al. (2016). Durable coexistence of donor and recipient strains after fecal microbiota transplantation. Science 352, 586-589. doi: 10.1126/science.aad8852

Manichanh, C., Reeder, J., Gibert, P., Varela, E., Llopis, M., Antolin, M., et al. (2010). Reshaping the gut microbiome with bacterial transplantation and antibiotic intake. Genome Res. 20, 1411-1419. doi: 10.1101/gr.107987.110

Mattila, E., Uusitalo-Seppälä, R., Wuorela, M., Lehtola, L., Nurmi, H., Ristikankare, M., et al. (2012). Fecal transplantation, through colonoscopy, is effective therapy for recurrent Clostridium difficile infection. Gastroenterology 142, 490-496. doi: 10.1053/j.gastro.2011.11.037 
Moayyedi, P., Surette, M. G., Kim, P. T., Libertucci, J., Wolfe, M., Onischi, C., et al. (2015). Fecal microbiota transplantation induces remission in patients with active ulcerative colitis in a randomized controlled trial. Gastroenterology 149, 102.e6-109.e6. doi: 10.1053/j.gastro.2015.04.001

Morotomi, M., Guillem, J. G., Pocsidio, J., LoGerfo, P., Treat, M., Forde, K. A., et al. (1989). Effect of polyethylene glycol-electrolyte lavage solution on intestinal microflora. Appl. Environ. Microbiol. 55, 1026-1028.

Narula, N., Kassam, Z., Yuan, Y., Colombel, J. F., Ponsioen, C., Reinisch, W., et al. (2017). Systematic review and meta-analysis: fecal microbiota transplantation for treatment of active ulcerative colitis. Inflamm. Bowel Dis. 23, 1702-1709. doi: $10.1097 / \mathrm{mib} .0000000000001228$

Oksanen, J., Blanchet, F., Friendly, M., Kindt, R., Legendre, P., McGlinn, D., et al. (2019). vegan: Community Ecology Package. R package version 2.5-5.

Pinheiro, J., Bates, D., DebRoy, S., Sarkar, D., and R Core Team (2019). nlme: Linear and Nonlinear Mixed Effects Models. $R$ package version 3.1-141.

Qualls, J. E., Kaplan, A. M., van Rooijen, N., and Cohen, D. A. (2006). Suppression of experimental colitis by intestinal mononuclear phagocytes. J. Leukoc. Biol. 80, 802-815. doi: 10.1189/jlb.1205734

Quast, C., Pruesse, E., Yilmaz, P., Gerken, J., Schweer, T., Yarza, P., et al. (2013). The SILVA ribosomal RNA gene database project: improved data processing and web-based tools. Nucleic Acids Res. 41, D590-D596. doi: 10.1093/nar/gks1219

Quraishi, M. N., Widlak, M., Bhala, N., Moore, D., Price, M., Sharma, N., et al. (2017). Systematic review with meta-analysis: the efficacy of faecal microbiota transplantation for the treatment of recurrent and refractory Clostridium difficile infection. Aliment. Pharmacol. Ther. 46, 479-493. doi: 10.1111/apt. 14201

Rossen, N. G., Fuentes, S., van der Spek, M. J., Tijssen, J. G., Hartman, J. H., Duflou, A., et al. (2015). Findings from a randomized controlled trial of fecal transplantation for patients with ulcerative colitis. Gastroenterology 149, 110.e4-118.e4. doi: 10.1053/j.gastro.2015.03.045

Salonen, A., Nikkilä, J., Jalanka-Tuovinen, J., Immonen, O., Rajilić-Stojanović, M., Kekkonen, R. A., et al. (2010). Comparative analysis of fecal DNA extraction methods with phylogenetic microarray: effective recovery of bacterial and archaeal DNA using mechanical cell lysis. J. Microbiol. Methods 81, 127-134. doi: 10.1016/j.mimet.2010.02.007

Santiago, A., Panda, S., Mengels, G., Martinez, X., Azpiroz, F., Dore, J., et al. (2014). Processing faecal samples: a step forward for standards in microbial community analysis. BMC Microbiol. 14:112. doi: 10.1186/1471-2180-14-112

Satokari, R., Mattila, E., Kainulainen, V., and Arkkila, P. E. (2015). Simple faecal preparation and efficacy of frozen inoculum in faecal microbiota transplantation for recurrent Clostridium difficile infection-an observational cohort study. Aliment. Pharmacol. Ther. 41, 46-53. doi: 10.1111/apt. 13009

Schuetz, A. N. (2014). Antimicrobial resistance and susceptibility testing of anaerobic bacteria. Clin. Infect. Dis. 59, 698-705. doi: 10.1093/cid/ciu395
Sorg, R. A., Lin, L., van Doorn, G. S., Sorg, M., Olson, J., Nizet, V., et al. (2016). Collective resistance in microbial communities by intracellular antibiotic deactivation. PLoS Biol. 14:e2000631. doi: 10.1371/journal.pbio.2000631

Staley, C., Kaiser, T., Beura, L. K., Hamilton, M. J., Weingarden, A. R., Bobr, A., et al. (2017). Stable engraftment of human microbiota into mice with a single oral gavage following antibiotic conditioning. Microbiome 5:87. doi: 10.1186/ s40168-017-0306-2

Staley, C., Kelly, C. R., Brandt, L. J., Khoruts, A., and Sadowsky, M. J. (2016). Complete microbiota engraftment is not essential for recovery from recurrent Clostridium difficile infection following fecal microbiota transplantation. MBio 7:e1965-16. doi: 10.1128/mBio.01965-16

van Nood, E., Vrieze, A., Nieuwdorp, M., Fuentes, S., Zoetendal, E. G., de Vos, W. M., et al. (2013). Duodenal infusion of donor feces for recurrent Clostridium difficile. N. Engl. J. Med. 368, 407-415. doi: 10.1056/NEJMoa1205037

Venables, W., and Ripley, B. (2002). Modern Applied Statistics with S. New York, NY: Springer.

Wang, R. F., Beggs, M. L., Robertson, L. H., and Cerniglia, C. E. (2002). Design and evaluation of oligonucleotide-microarray method for the detection of human intestinal bacteria in fecal samples. FEMS Microbiol. Lett. 213, 175-182. doi: 10.1016/s0378-1097(02)00802-9

Wos-Oxley, M. L., Bleich, A., Oxley, A. P., Kahl, S., Janus, L. M., Smoczek, A., et al. (2012). Comparative evaluation of establishing a human gut microbial community within rodent models. Gut Microbes 3, 234-249. doi: 10.4161/gmic. 19934

Wrzosek, L., Ciocan, D., Borentain, P., Spatz, M., Puchois, V., Hugot, C., et al. (2018). Transplantation of human microbiota into conventional mice durably reshapes the gut microbiota. Sci. Rep. 8:6854. doi: 10.1038/s41598-018-25300-3

Xiao, L., Feng, Q., Liang, S., Sonne, S. B., Xia, Z., Qiu, X., et al. (2015). A catalog of the mouse gut metagenome. Nat. Biotechnol. 33, 1103-1108. doi: 10.1038/nbt. 3353

Xu, D., Chen, V. L., Steiner, C. A., Berinstein, J. A., Eswaran, S., Waljee, A. K., et al. (2019). Efficacy of fecal microbiota transplantation in irritable bowel syndrome: a systematic review and meta-analysis. Am. J Gastroenterol. 114, 1043-1050. doi: 10.14309/ajg.0000000000000198

Conflict of Interest: The authors declare that the research was conducted in the absence of any commercial or financial relationships that could be construed as a potential conflict of interest.

Copyright (C) 2019 Freitag, Hartikainen, Jouhten, Sahl, Meri, Anttila, Mattila, Arkkila, Jalanka and Satokari. This is an open-access article distributed under the terms of the Creative Commons Attribution License (CC BY). The use, distribution or reproduction in other forums is permitted, provided the original author(s) and the copyright owner(s) are credited and that the original publication in this journal is cited, in accordance with accepted academic practice. No use, distribution or reproduction is permitted which does not comply with these terms. 